\title{
Fluoride Inhibition in Patients with Atypical Dibucaine-Resistant Plasmacholinesterase ${ }^{1}$
}

\author{
Sarla P. Kothary, ${ }^{2}$ Kathleen B. Flynn, ${ }^{2}$ and Elemér K. Zsigmond ${ }^{2}$ \\ Received 5 Jan. 1979-Final 11 May 1979
}

The fluoride numbers ( $F N$ ) of 144 individuals referred to our laboratory for dibucaine number $(D N)$ determinations because of prolonged apnea following succinylcholine in the probands or relatives were determined by our new method using $2.5 \times 10^{-4} \mathrm{M}$ concentration of sodium fluoride at $37 \mathrm{C}$. Dibucaine-resistant homozygotes and heterozygotes both had lower FN than the normal homozygotes. A linear correlation between $D N$ and $F N$ for all these genotypes was found which is described by the equation $F N=0.59 D N+32$. The correlation coeffcient was $r=0.94$. This equation will help in the correct identification of atypical fluoride resistant genotypes, since these ought to have an observed $F N$ lower by 2 standard deviations than the FN calculated from this equation.

KEY WORDS: FN-DN correlation; fluoride-resistant plasmacholinesterase, dibucaine-resistant plasmacholinesterase.

\section{INTRODUCTION}

Genetic anomalies of the dibucaine-resistant $\left(E_{l}^{a} E_{l}^{a}\right)$, the fluoride-resistant $\left(E_{j}^{f} E_{j}^{f}\right)$, and the silent $\left(E_{j}^{s} E_{j}^{\dagger}\right)$ homozygous plasmacholinesterase genotypes are known to be responsible for the prolonged paralysis of the respiratory muscles (prolonged apnea) and cardiac arrhythmias following the intravenous injection of the usual $1.0 \mathrm{mg} / \mathrm{kg}$ dose of succinylcholine, the most widely used

\footnotetext{
'A preliminary report was presented at the American Society of Anesthesiologists" annual meeting. October 7-11, 1973.

${ }^{2}$ University of Michigan Medical Center, Ann Arbor, Michigan 48109.
} 
muscle relaxant during anesthesia and surgery. The recognition of this anomaly and the genetic survey of the index family after the identification of the proband may circumvent untoward reactions to succinylcholine in the affected relatives (Harris and Whittaker, 1961; Foldes et al., 1963).

Since the discovery of the fluoride-resistant plasmacholinesterase, many cases of fluoride-resistant heterozygotes $\left(E_{l}^{u} E_{l}\right)$ and only a few homozygotes $\left(E_{l}^{f} E_{l}^{f}\right)$ have been reported (Harris and Whittaker, 1961; Whittaker, 1967, 1968; Foldes et al., 1963; Whittaker and Vickers, 1970; Thompson and Whittaker, 1966). In all these studies $\mathrm{Na}$ fluoride at a concentration of $5.0 \times 10^{-5} \mathrm{M}$ was used, which was claimed to inhibit normal plasma cholinesterase to about $64 \%$ while the fluoride-resistant homozygote was inhibited to less than $34 \%$. Some of the reports on fluoride-resistant genotypes lacked the determination of $\mathrm{DN}$, although some reported dibucaine numbers were also found abnormal in the so-called fluoride-resistant homozygotes and fluorideresistant-silent heterozygotes. Moreover, the determination of the fluoride number (FN) alone could not differentiate between fluoride-dibucaine-resistant heterozygotes and fluoride-resistant homozygotes. The latter could only be differentiated by a normal DN.

The narrow range of percentages $(64-34 \%$ ) of inhibition in the various genotypes with the use of $5.0 \times 10^{-5} \mathrm{M} \mathrm{Na}$ fluoride concentration at $25 \mathrm{C}$ in contrast to the wide separation with the same concentration of dibucaine $(15-75 \%)$ necessitated the development of a more specific method for the differentiation of the fluoride resistant phenotypes (Zsigmond, 1973). Because of the great dependence of the fluoride inhibition on temperature, we also increased the temperature from 25 to $37 \mathrm{C}$ and increased the concentration of $\mathrm{Na}$ fluoride to $2.5 \times 10^{-4} \mathrm{M}$, which gives $75 \%$ inhibition in a normal homozygote. We have been utilizing this modified method during the past 5 years to determine whether the fluoride inhibition may also be dependent on dibucaine inhibition. Therefore, both DN and FN were determined in a large number of dibucaine-resistant genotypes $\left(E_{I}^{u} E_{I}^{a}\right.$ and $\left.E_{I}^{a} E_{I}^{a}\right)$.

\section{MATERIALS AND METHODS}

Between 1973 and 1978 a total of 144 plasma samples of patients with a history of prolonged apnea following succinylcholine administration and their blood relatives were sent to our laboratory for the determination of DN and FN.

The samples were stored at $-20 \mathrm{C}$ until the time of determination. All the experiments were carried out at $37 \mathrm{C}$ and $p \mathrm{H}$ 7.4. Benzoylcholine chloride at a concentration of $5.0 \times 10^{-5} \mathrm{M}$ was used as a substrate for the determination of enzyme activity. Nupercaine hydrochloride at a concentration of $1.0 \times 10^{-5} \mathrm{M}$ and $\mathrm{Na}$ fluoride at a concentration of $2.5 \times 10^{-4} \mathrm{M}$ were used as inhibitors for the determination of $\mathrm{DN}$ and $\mathrm{FN}$, respectively. All determinations were 
carried out by a modification (Foldes et al., 1960) of Kalow's ultraviolet spectrophotometric method using Beckman DU spectrophotometer at the wavelength of $240 \mathrm{~nm}$.

\section{RESULTS AND DISCUSSION}

As shown in Table I, 50 individuals were normal homozygotes with mean $\pm \mathrm{SD}$ $\mathrm{DN}$ of $76.0 \pm 2.7$ and $\mathrm{FN}=77.0 \pm 3.2$. Fifty-four individuals were atypical normal heterozygotes with mean \pm SD DN of $59.0 \pm 7.4$ and $F N=67.0 \pm 5.4$. The remaining 40 individuals were atypical dibucaine-resistant homozygotes with means \pm SD DN of $18.0 \pm 7.7$ and $F N=43.0 \pm 10.0$. It is evident that the dibucaine-resistant heterozygotes and homozygotes also have abnormal fluoride inhibition or FN. There is a linear correlation between DN and FN with the equation shown in Fig. $1(r=0.94)$. So far we have not observed any plasma sample where FN would be 2 SD lower than DN. Consequently we were unable to identify any fluoride resistant heterozygote or homozygote, while we observed the expected incidence of dibucaine-resistant silent and usual genotypes in a surgical and anesthetized patient population (Whittaker and Vickers, 1970).

The original reports on the fluoride-resistant genotypes indicated a correlation between this genotype and prolonged apnea following succinylcholine administration (Harris and Whittaker, 1961; Whittaker, 1967; Whittaker and Vickers, 1970). Because of the paucity of the reported fluoride-resistant homozygotes with adequately documented prolonged apnea (Whittaker and

Table I. Correlation of DN with FN in Apneic Patients and Relatives With Various Plasmacholinesterase Genotypes

\begin{tabular}{lrrr}
\hline Genotype & \multicolumn{1}{c}{$\begin{array}{c}\text { BeCh } \\
\mathrm{HR}^{a}\end{array}$} & \multicolumn{1}{c}{$\mathrm{DN}$} & \multicolumn{1}{c}{ FN } \\
\hline$E_{l}^{u} E_{l}^{u}$ & 86.62 & $76.0 \pm 2.68$ & $77.0 \pm 3.22$ \\
$N=50$ & $\pm 30.06^{b}$ & \pm 0.38 & \pm 0.45 \\
& $\pm 4.25^{c}$ & & \\
$E_{l}^{a} E_{l}^{u}$ & 55.99 & $59.0 \pm 7.41$ & $67.0 \pm 5.37$ \\
$N=54$ & \pm 23.59 & \pm 1.00 & \pm 0.73 \\
& \pm 3.21 & & \\
$E_{l}^{a} E_{l}^{a}$ & 29.80 & $18.0 \pm 7.66$ & $43.0 \pm 10.05$ \\
$N=40$ & \pm 11.71 & \pm 1.21 & \pm 1.59 \\
& \pm 1.88 & & \\
\hline
\end{tabular}

\footnotetext{
${ }^{a}$ Benzoylcholine hydrolysis expressed as $\mu \mathrm{mol} / \mathrm{ml} / \mathrm{hr}$.

${ }^{\mathrm{b}}$ Standard deviation.

c Standard error.
} 


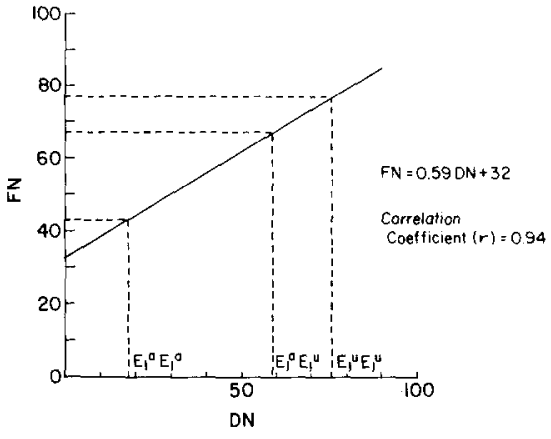

Fig. 1. Correlation of $F N$ with $D n$ in various dibucaine-resistant genotypes. Note that the FN correlates linearly with the $\mathrm{DN}$ in all genotypes with a correlation coefficient of $r=0.94$. This equation can be utilized to predict the expected $\mathrm{Fn}$ in a dibucaine-resistant genotype. The observed FN should be lower by 2 SD deviation in a fluoride-resistant genotype than the value predicted from this equation.

Vickers, 1970) and because of the occurrence of prolonged apnea even in a large number of $E_{l}^{u} E_{l}^{u}$ (Foldes et al., 1963; Zsigmond et al., 1973), it is difficult to tell the clinical importance of fluoride-resistant genotypes. The only objective scientific way to establish a correlation between the abnormal genotype and prolonged neuromuscular block induced by succinylcholine is to determine the duration of neuromuscular block in patients with the fluorideresistant PChE following intravenous succinycholine infusion.

\section{CONCLUSION}

The reported correlation equation will, it is hoped, facilitate the correct identification of the fluoride-resistant genotypes of PChE, thereby avoiding erroneous reporting of these genotypes in the future.

\section{REFERENCES}

Foldes, F. F., Erdos, E. G., Zsigmond, E. K., and Zwartz, J. A. (1960). Reactivation of neostigmine inhibited human plasma cholinesterase. J. Pharmacol. Exp. Ther. 129:394.

Foldes, F. F., Foldes, V. M., Smith, J. C., and Zsigmond, E. K. (1963). The relation between plasma cholinesterase and prolonged apnea cause by succinylcholine. Anesthesiology 24:208.

Harris, H., and Whittaker, M. (1961). Differential inhibition of human serum cholinesterase with fluoride: Recognition of two new phenotypes. Nature 191:496.

Thompson, J. C., and Whittaker, M. (1966). A study of the pseudocholinesterase in 78 cases of apnea following suxamethonium. Acta Genet. 16:209.

Whittaker, M. (1967). The pseudocholinesterase variants: A study of fourteen families selected via the fluoride resistant phenotype. Acta Genet. Stat. Med. 17:1.

Whittaker, M. (1968). The pseudocholinesterase variants: Esterase levels and increased resistance to fluoride. Acta Genet. Stat. Med. 18:325.

Whittaker, M., and Vickers, M.D. (1970). Initial experiences with the cholinesterase research unit. Br. J. Anaesthesiol. 42:1016.

Zsigmond, E.K. (1973). Fluoride numbers. Letter to the Editor, Br. J. Anesthesiol. 45:1053.

Zsigmond, E. K., Flynn, K. B., Matsuki, A., Kothary, S. P., M.B., Eilderton, T. E., D.D.S., D.M.D., Robins, G., D.D.S., and Downs, J. R., D.D.S. (1973). Atypical plasmacholinesterase as a cause of prolonged apnea 10 years experience with plasma cholinesterase laboratory. Abstracts of Papers, ASA Annual Meeting, October 7-11. 